\title{
Optimal Capacity Allocation when Patients encounter Congestion in Primary Healthcare Network
}

\author{
Kaushal Kumar \\ Department of Operational Research \\ Faculty of Mathematical Sciences \\ University of Delhi, Delhi-110007, India \\ E-mail: kkumar@or.du.ac.in
}

(Received November 1, 2018; Accepted December 15, 2018)

\begin{abstract}
Universal health care aims at providing low cost or if possible free primary care to everyone. Most countries pursue this goal and it is pertinent for developing countries to make the best use of their limited resources to achieve it. In spite of every effort from the government, unfortunately patients in India spend significant amount of money on travelling and out-of-pocket expenses for availing primary care services even at public funded facilities. We propose an optimization model to help health decision makers in managing existing capacity for alleviation of this problem. The model can be used in the identification of existing health care facilities that need to be upgraded or reduced with a view to improve their utilization at minimum cost. The model recognizes increase in patient out-of-pocket expenses incurred at facilities due to longer waiting time (congestion). Results from numerical experiments are presented to explicate the functioning of the model.
\end{abstract}

Keywords- Primary care, Public Health, Waiting time, Optimization, Out-of-pocket expense.

\section{Introduction}

Public health care services in developing countries need improvement. Patients often skip nearby subsidized services of government run health centres and visit costly privately managed health care facilities (Rao and Sheffel, 2018). This uneven utilization does not augur well with the limited capacities of public health systems of these countries. Due to dismal efficiency in deployment of resources, countries like India fare poorly under every community health indicators. The health care financing system may also be contributing to inequity in health care and low quality health services (Kumar et al., 2011). Economically poorer patients, who are the most vulnerable, should be protected against burden of health care expenses (Sohrabi and Tumin, 2016). Government initiatives like building physical infrastructure can be carried out through more investments but deploying considerable number of trained physicians in quick time is almost impossible. It is therefore required to make investments judiciously to manage and employ the existing limited capacity in an optimal manner. Moreover, policy-making in healthcare could be improved by capturing big data using information technology (Wang and Alexander, 2019). Wang(2018) discussed the importance of big data analytics for the extraction of hidden information, which may be invaluable for policy-making.

Primary health care is both curative and conducive. It can serve greater number of people at lower cost and reduce the burden on tertiary care systems. The government provides free primary care through primary health centres (PHCs) and community health centres (CHCs). It is often observed that PHCs and CHCs are unevenly utilized, some are sparsely used and many are over loaded. There is also discouraging perception about the quality of care provided at these facilities. Many patients especially those from financially weaker background approach costly private 
International Journal of Mathematical, Engineering and Management Sciences

Vol. 4, No. 2, 400-408, 2019

https://dx.doi.org/10.33889/IJMEMS.2019.4.2-032

centres for availing primary health care (Sangar et al., 2018). In the process, the patients can make recurring expenses adding to their misery (Balarajan et al., 2011). The out-of-pocket expense incurred on health care amounts for a significant percentage of entire household expenditure (Garg and Karan, 2008) and it is one of the primary reasons behind pushing many household further towards poverty (Hamid et al., 2014).

Public funded facilities are confronted with various other problems too, viz. shortage of infrastructure, inadequate medicines and limited medical staff etc. (Agarwal et al., 2017). The health care system is therefore required to undergo transformations in order to improve efficiency and standards of service (Hamid et al., 2014). Saksena et al. (2010) have found that consultation fees at public facilities may not be a bigger component of out-of-pocket expense and expense on medicines, compulsion to use private facilities, transportation cost etc. are sizable segments. These components need to be considered while developing improvement strategies.

The primary health centres are initial point of connection between the patients and medical staff. The current condition of primary care health centres is miserable and redevelopment of existing facilities is required along with acquiring new resources. It is pertinent to revaluate every public facility for its patient attracting potential. Attractiveness of a facility could be enhanced by locating it near localities and providing health care service aimed at reducing out-of-pocket expenses incurred by the patients. Facilities with lesser footfall should be downgraded and the excess capacity may be re-assigned for improving utilization. Some research publications have suggested ways to find optimal policies in this regard. Antunes and Peeters (2001) discussed a multi-period facility location model for restructuring the existing network of facilities by opening some new facilities, closure of some existing facilities and simultaneously broadening and shrivelling existing facilities. Jena et al. (2016) talked about location problems involving capacity-modification of existing network of facilities. Araya et al.(2012) proposed school network planning model, where some existing schools can be closed.

A substantial disparity is observed between capacity of government health care centres and the demand for health care. This gap leads to overcrowded public facilities in the system and therefore majority of public facilities experience congestion. Zhang et al. (2009) proposed a healthcare facility location model incorporating congestion. Davari et al. (2016) suggested a mixed-integer programming model to design a health care network under budget and congestion considerations and also proposed a heuristic approach for solving the proposed model. A congested facility is unable to provide health care services to every patient and the patients are compelled to go elsewhere to get the services. The out-of-pocket expense therefore increases when there is higher waiting time for patients. This idea is central to the proposed model of this article. Majority of location-allocation modelling literature has not explicitly considered out-ofpocket expense incurred by the patients. The model proposed in this article addresses this gap and also includes congestion costs at facilities.

In our optimization model, the objective is to minimize the total costs to patients through improvement of the utilization of public funded health care facilities. The proposed model is thus an attempt to determine those public funded health care facilities with limited potential requiring degradation and also the facilities requiring expansion. In the next section, the optimization model is developed. In section-3 results from numerical experiments and their implications are presented. 
International Journal of Mathematical, Engineering and Management Sciences

Vol. 4, No. 2, 400-408, 2019

https://dx.doi.org/10.33889/IJMEMS.2019.4.2-032

\section{Model Development}

Consider a region where primary health care services are delivered through facilities of two types: primary care facilities or primary health centres (PHCs) and secondary care facilities or community health centres (CHCs). PHCs provide basic medical care and do not include any inpatient services whereas CHCs are larger facilities and they provide additional services like diagnostic and specialist services. Patients visiting these facilities to avail health care services are supposed to incur travelling cost and cost of treatment. Although the treatment at government facilities is absolutely free, in spite of that a patient may incur direct and indirect costs for purchasing medicines, medical tests, waiting in queue and many more. We assume that the outof-pocket spending at PHCs is more than those at CHCs. We also assume that the out-of-pocket expense incurred at public facilities depend on the number of patients visiting the facilities. If we observe congestion at some public facility, then the out-of-pocket expense incurred by the patient is bound to increase, due to unproductive waiting time. Let us define $\alpha$ as the threshold value such that if number of patients at the facility exceeds $\alpha$, then there is congestion and patient expense incurred at the facility increases. This is modelled as following pair of conditions:

$$
\begin{array}{ll}
\text { If } \quad \sum_{i \in I} P_{i} X_{i j}^{k} \leq \alpha, \text { then } E_{j}^{k}=L_{j}^{k} & \forall j \in J, k \in K \\
\text { If } \quad \sum_{i \in I} P_{i} X_{i j}^{k}>\alpha \text {, then } E_{j}^{k}=H_{j}^{k} & \forall j \in J, k \in K .
\end{array}
$$

$P_{i}$ represents demand for primary health care services from population zone $i \in I . X_{i j}^{k}$ is the proportion of the population in locality $i \in I$ visiting a type $k \in K$ facility at site $j \in J$ for getting primary health care services. $E_{j}^{k}$ is the out-of-pocket expense incurred at a facility of type $k$ located at site $j$. This out-of-pocket expense takes the value $L_{j}^{k}$ when the number of patients visiting the facility does not exceed the capacity of the facility while out-of-pocket expense increases and becomes $H_{j}^{k}$ if the facility experiences congestion and facility is overused. The either-or conditions given above can be dealt with by defining an auxiliary binary variable $W_{j}^{k}$ as follows

$$
\begin{array}{ll}
E_{j}^{k}=L_{j}^{k}\left(1-W_{j}^{k}\right)+H_{j}^{k}\left(W_{j}^{k}\right) & \forall j \in J, k \in K \\
\sum_{i \in I} P_{i} X_{i j}^{k} \leq \alpha+N\left(W_{j}^{k}\right) & \forall j \in J, k \in K \\
\sum_{i \in I} P_{i} X_{i j}^{k}>\alpha\left(W_{j}^{k}\right) & \forall j \in J, k \in K
\end{array}
$$

$N$ is a very large number. When $W_{j}^{k}$ takes the value 0 , there is no congestion at type $k \in K$ facility at site $j \in J$ and out-of-pocket expense incurred is $L_{j}^{k}$, whereas $W_{j}^{k}$ taking the value 1 implies there is congestion at type $k \in K$ facility at site $j \in J$ and out-of-pocket expense increases to $H_{j}^{k}$ as a result. 
International Journal of Mathematical, Engineering and Management Sciences

Vol. 4, No. 2, 400-408, 2019

https://dx.doi.org/10.33889/IJMEMS.2019.4.2-032

The decision-making problem that is proposed may facilitate the policy maker in improving the utilization of government facilities and simultaneously brings down the out-of-pocket expense incurred by the patients. The proposed model tries to find the number of PHCs to be upgraded to higher level CHCs and it may also degrade the existing CHCs so that excess resources could be utilized elsewhere. Capacity management is done in such a way that congestion at some facility should be avoided by means of up-gradation and simultaneously de-gradation of appropriate facilities should be performed to improve utilization. For the given decision problem, we propose the following set covering optimization model

Minimize $\sum_{i \in I} P_{i} \sum_{j \in J} \sum_{k \in K}\left(C_{i j}+E_{j}^{k}\right) X_{i j}^{k}$

Subject to

$$
\begin{array}{lc}
Y_{j}^{p} \leq 2-\left(M_{j}+N_{j}+U_{j}\right) & \forall j \in J \\
1-Y_{j}^{p} \leq\left(M_{j}+N_{j}-D_{j}\right) & \forall j \in J \\
Y_{j}^{c} \leq 2-\left(M_{j}+N_{j}+D_{j}\right) & \forall j \in J \\
1-Y_{j}^{c} \leq\left(M_{j}+N_{j}-U_{j}\right) & \forall j \in J \\
1-Y_{j}^{p} \geq\left(N_{j}-D_{j}\right) & \forall j \in J \\
1-Y_{j}^{c} \geq\left(M_{j}-U_{j}\right) & \forall j \in J \\
U_{j}+D_{j} \leq 1 & \forall j \in J \\
Y_{j}^{p}+Y_{j}^{c}=1 & \forall j \in J \\
M_{j}+N_{j}=1 & \forall j \in J \\
U_{j} \leq M_{j} & \forall j \in J \\
D_{j} \leq N_{j} & \forall j \in J \\
X_{i j}^{k} \leq Y_{j}^{k} & \forall j \in J \\
C_{j}^{u} \sum_{j \in J} U_{j}+C_{j}^{d} \sum_{j \in J} D_{j} \leq B & \\
\sum_{j \in J} U_{j}=\sum_{j \in J} D_{j} & \forall j \in J \\
\sum_{j \in J} \sum_{k \in K} X_{i j}^{k}=1 & \forall i \in I \\
M_{j}, N_{j}, U_{j}, D_{j} \in[0,1] & \forall j \in J \\
Y_{j}^{c}, Y_{j}^{p} \in\{0,1\} & \forall j \in J \\
X_{i j}^{k} \geq 0 &
\end{array}
$$


International Journal of Mathematical, Engineering and Management Sciences

Vol. 4, No. 2, 400-408, 2019

https://dx.doi.org/10.33889/IJMEMS.2019.4.2-032

The notations used in the model are as follows: $I, J$ represent the set of all population zones and sites of government facilities respectively. $K$ represents the type of government facility, i.e. $K=$ $\{P H C, C H C\} . C_{i j}$ is the travelling cost from population zone $i \in I$ to facility at site $j \in$ J. $C_{j}^{u}$ and $C_{j}^{d}$ are costs incurred on upgrading a PHC and downgrading a $\mathrm{CHC}$ respectively at location $j \in J . B$ is government budget for the planning horizon. In the current network of facilities, $M_{j}$ and $N_{j}$ specify the existence of a PHC, CHC respectively at location $j \in J$. The variables in the model are: $U_{j}$ and $D_{j}$ are binary variables depicting the up-gradation of a PHC and de-gradation of a $\mathrm{CHC}$ respectively at location $j \in J$. There are binary variables, viz. $Y_{j}^{p}$ and $Y_{j}^{c}$, which denote the existence of a PHC and a $\mathrm{CHC}$ respectively at site $j \in J$ in the proposed network of facilities.

The objective function (4) minimizes the total out-of-pocket expenditure incurred by the patients seeking primary health care services at PHCs and CHCs. The PHCs which require up-gradation and the CHCs requiring de-gradation will be determined by constraints (5) - (10). Constraints (11-15) determine appropriate facilities for up-gradations and de-gradations. Contingency condition is given by constraint (16) and constraint (17) defines the budget restriction. Constraint (18) is forcing the number of up-gradations to be equal to number of de-gradations and constraint (19) is the covering constraint.

\section{Numerical Experiment}

This section explains the functioning of proposed optimization model and presents allocation results arising from optimization. It is assumed that there are ten population centres and ten operating public health care facilities, among them six are PHCs and four are CHCs. Table 1 contains population data for population centres and the travelling cost data (in INR) for travelling to respective facilities. The per patient out-of-pocket expense incurred at the facilities is driven by congestion and it is assumed to be INR 200 and INR 150 respectively for PHC and CHC during normal operations. The same cost increases to INR 300 and INR 225 for PHCs and CHCs respectively when there is congestion at these facilities. The up-gradation and de-gradation costs of PHCs and CHCs are assumed to be INR 10,00,000 and INR zero respectively.

Table 1. Population and travel cost data

\begin{tabular}{|c|c|c|c|c|c|c|c|c|c|c|c|}
\hline \multirow{2}{*}{$\begin{array}{c}\text { Population } \\
\text { centre }\end{array}$} & $\begin{array}{c}\text { Population } \\
\text { Figures }\end{array}$ & \multicolumn{10}{|c|}{ Facility sites (Travel cost in INR) } \\
\cline { 3 - 13 } & H1 & H2 & H3 & H4 & H5 & H6 & H7 & H8 & H9 & H10 \\
\hline P1 & 126 & 30 & 40 & 50 & 30 & 50 & 40 & 40 & 50 & 50 & 20 \\
\hline P2 & 84 & 60 & 40 & 50 & 40 & 50 & 40 & 60 & 30 & 50 & 60 \\
\hline P3 & 156 & 50 & 30 & 20 & 60 & 60 & 20 & 60 & 20 & 50 & 50 \\
\hline P4 & 118 & 20 & 20 & 30 & 40 & 20 & 20 & 50 & 40 & 60 & 40 \\
\hline P5 & 172 & 30 & 30 & 50 & 60 & 20 & 50 & 30 & 50 & 40 & 20 \\
\hline P6 & 72 & 20 & 20 & 40 & 40 & 50 & 20 & 50 & 50 & 20 & 20 \\
\hline P7 & 184 & 40 & 20 & 30 & 60 & 30 & 30 & 50 & 40 & 50 & 30 \\
\hline P8 & 144 & 20 & 30 & 30 & 60 & 40 & 40 & 20 & 30 & 50 & 40 \\
\hline P9 & 168 & 20 & 40 & 20 & 30 & 50 & 50 & 40 & 50 & 50 & 60 \\
\hline P10 & 102 & 60 & 60 & 30 & 50 & 40 & 40 & 50 & 60 & 50 & 40 \\
\hline
\end{tabular}


International Journal of Mathematical, Engineering and Management Sciences

Vol. 4, No. 2, 400-408, 2019

https://dx.doi.org/10.33889/IJMEMS.2019.4.2-032

An experiment is conducted to examine the influence of change in budget on model performance. It was assumed that congestion at a PHC takes place when it registers the arrival of more than 40 patients whereas a CHC is supposed to experience congestion if the arrival is more than 60 patients. Figure 1 represents the coverage by the health centres against budget and an increase in budget results in a slight increase in coverage by PHCs and slight decrease in coverage by CHCs and further budget increase decreases coverage of PHCs and increases the coverage of CHCs. This is happening due to increase in out-of-pocket expense incurred at PHCs and decrease in outof-pocket expense incurred at CHCs as shown in Figure 2. It is also observed that total out-ofpocket expense decreases from INR 3,06,850 to INR 3,06,650 with increase in budget from INR $10,00,000$ to 20,00,000 because number of up-gradations of PHCs to CHCs increases from 1 to 2 and a further increase in budget does not change the total out-of-pocket expense and number of up-gradations. None of the PHCs was found to be congested at all levels of budget whereas one $\mathrm{CHC}$ was congested which resulted in an increase in out-of-pocket expense at that $\mathrm{CHC}$.

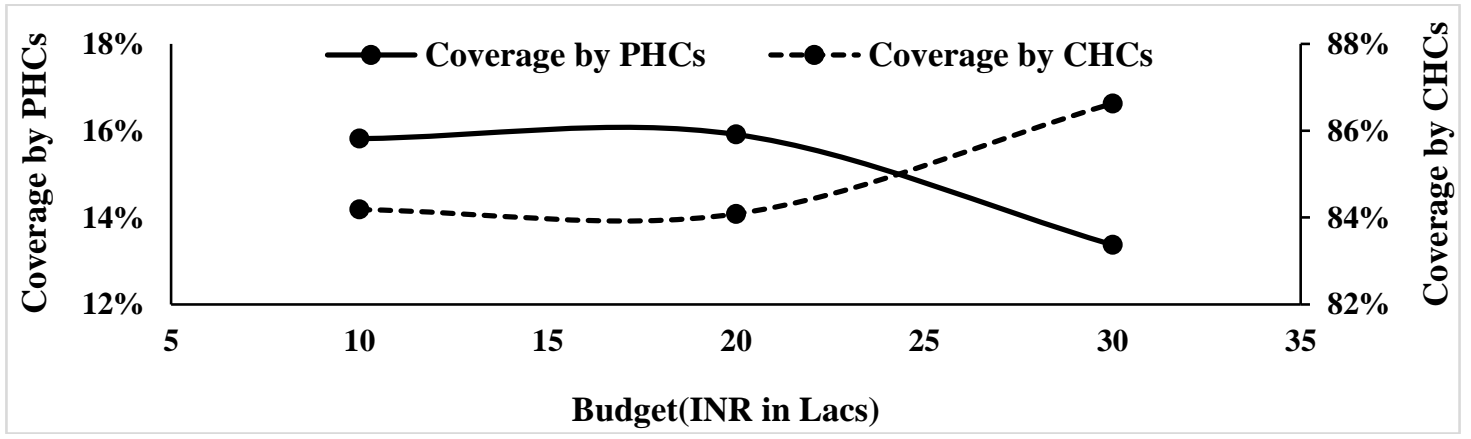

Figure 1. Budget vs. coverage

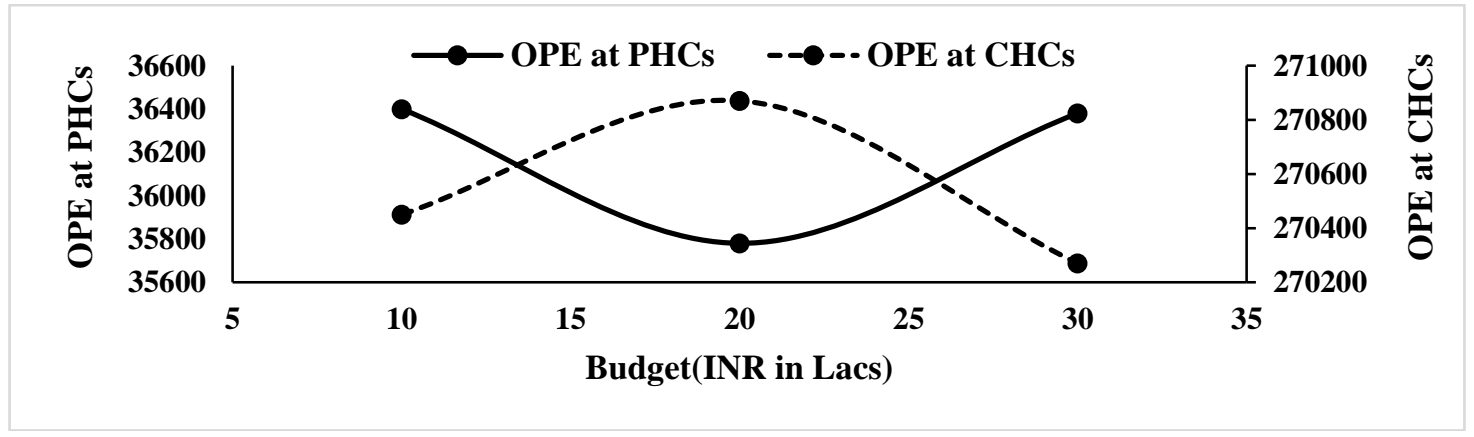

Figure 2. Budget vs. out-of-pocket expense (OPE)

Another experiment was conducted to see the influence of change in congestion point on model performance. In this experiment, we vary the threshold $\alpha$ (defined in section 2) for PHCs and CHCs simultaneously. Different levels of congestion for PHCs are considered to be 0, 20, 40, 60, 
International Journal of Mathematical, Engineering and Management Sciences

Vol. 4, No. 2, 400-408, 2019

https://dx.doi.org/10.33889/IJMEMS.2019.4.2-032

80 and 100, whereas for CHCs the congestion points are considered as $0,30,60,90,120,150$ for this experiment. Budget has been fixed at INR 20,00,000. Figures 3 and 4 show the impact of change in congestion points of PHCs and CHCs on coverage and out-of-pocket expense respectively. At points 1, 2, 3, 4, 5 and 6 on the horizontal axis in Figures 3 and 4, the congestion points of PHCs and CHCs are $(0,0),(20,30),(40,60),(60,90),(80,120)$ and $(100,150)$ respectively. As shown in Figure 3, an increase in the level of congestion results in an increase in coverage of PHCs and decrease in coverage of CHCs which means PHCs are now accommodating more patients which is, therefore, increasing the out-of-pocket expense at PHCs as seen in Figure 4. This finding possibly implies that an increase in the capacity of PHCs assists in reducing the workload of higher level CHCs. Moreover, out-of-pocket expense incurred at the PHCs is experiencing an increase due to the arrival of more patients but the overall out-of-pocket expenditure incurred by the population is declining as seen in Figure 4.

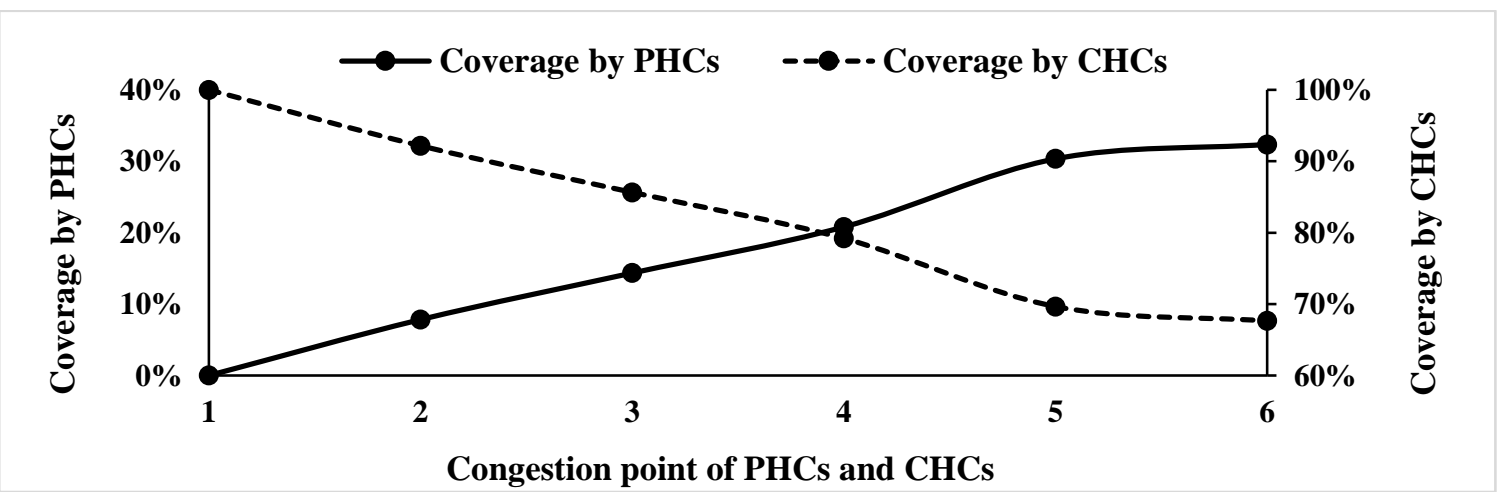

Figure 3. Congestion level vs. coverage

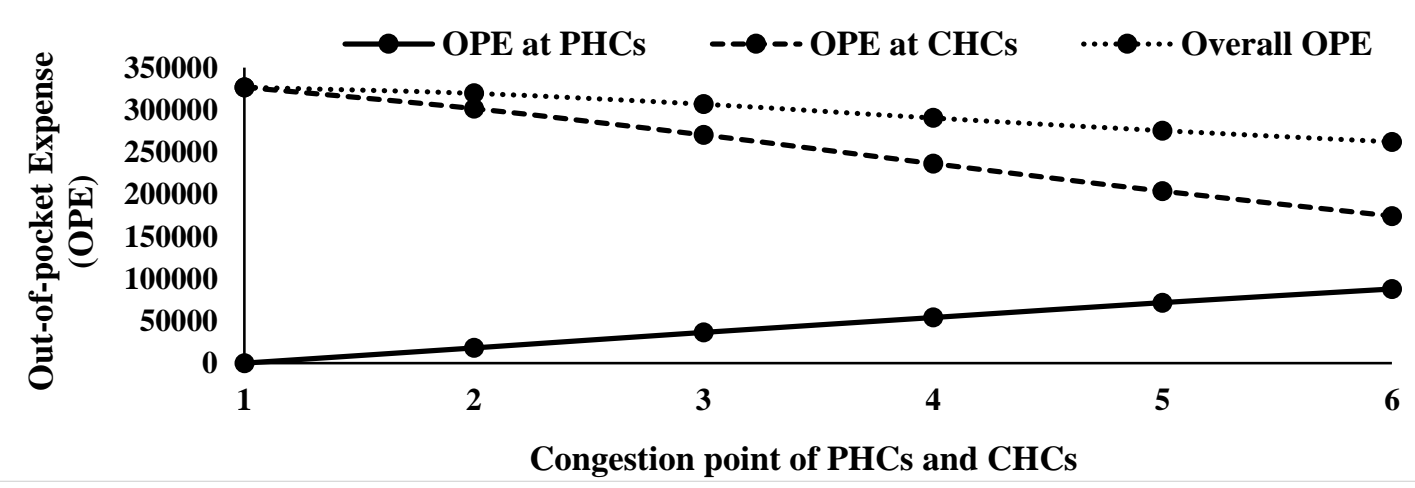

Figure 4. Congestion level vs. out-of-pocket expense (OPE) 
International Journal of Mathematical, Engineering and Management Sciences

Vol. 4, No. 2, 400-408, 2019

https://dx.doi.org/10.33889/IJMEMS.2019.4.2-032

\section{Conclusion}

A social problem of rising out-of-pocket expenses towards primary health care services is considered in this article. Policy makers on health care could use the proposed model in strategic management of existing capacity. In location-allocation modelling literature, the concept of patient out-of-pocket expense along with congestion cost is included for perhaps the first time to the best of our knowledge. Numerical experiments have been conducted to explain the working of the model and the same could be used by the decision makers. The proposed model is a mixed integer quadratic programming (MIQP) problem and most MIQP problems are NP-hard which require solution algorithms to deal with larger instances of the problem. Some articles on solution methods are: Nyamugure et al. (2017), Al-Hasani et al. (2018), Turgay (2018). In future, new formulations of congestion cost based on queuing theoretic models would be developed.

\section{Conflict of Interest}

The author declares that there is no conflict of interest in this publication.

\section{Acknowledgement}

The author would like to express his sincere thanks to the Editor-in-Chief for his valuable suggestions towards improvisation of the article.

\section{References}

Agarwal, R., Jain, P., Ghosh, M. S., \& Parihar, K. S. (2017). Importance of primary health care in the society. International Journal of Health Sciences, 1(1), 6-11.

Al-Hasani, A., Al-Rabeeah, M., Kumar, S., \& Eberhard, A. (2018). An improved CPU time in triangle splitting method for solving a biobjective mixed integer program. International Journal of Mathematical, Engineering and Management Sciences, 3(4), 351-364.

Antunes, A., \& Peeters, D. (2001). On solving complex multi-period location models using simulated annealing. European Journal of Operational Research, 130(1), 190-201.

Araya, F., Dell, R., Donoso, P., Marianov, V., Martínez, F., \& Weintraub, A. (2012). Optimizing location and size of rural schools in Chile. International Transactions in Operational Research, 19(5), 695-710.

Balarajan, Y., Selvaraj, S., \& Subramanian, S. V. (2011). Health care and equity in India. The Lancet, 377(9764), 505-515.

Davari, S., Kilic, K., \& Naderi, S. (2016). A heuristic approach to solve the preventive health care problem with budget and congestion constraints. Applied Mathematics and Computation, 276, 442-453.

Garg, C. C., \& Karan, A. K. (2008). Reducing out-of-pocket expenditures to reduce poverty: a disaggregated analysis at rural-urban and state level in India. Health Policy and Planning, 24(2), 116128.

Hamid, S. A., Ahsan, S. M., \& Begum, A. (2014). Disease-specific impoverishment impact of out-ofpocket payments for health care: evidence from rural Bangladesh. Applied health Economics and Health Policy, 12(4), 421-433.

Jena, S. D., Cordeau, J. F., \& Gendron, B. (2016). Solving a dynamic facility location problem with partial closing and reopening. Computers \& Operations Research, 67, 143-154. 
International Journal of Mathematical, Engineering and Management Sciences

Vol. 4, No. 2, 400-408, 2019

https://dx.doi.org/10.33889/IJMEMS.2019.4.2-032

Kumar, A. S., Chen, L. C., Choudhury, M., Ganju, S., Mahajan, V., Sinha, A., \& Sen, A. (2011). Financing health care for all: challenges and opportunities. The Lancet, 377(9766), 668-679.

Nyamugure, P., Munapo, E., Lesaoana, M., \& Kumar, S. (2017). A heuristic for a mixed integer program using the characteristic equation approach. International Journal of Mathematical, Engineering and Management Sciences, 2(1), 1-16.

Rao, K. D., \& Sheffel, A. (2018). Quality of clinical care and bypassing of primary health centers in India. Social Science \& Medicine, 207, 80-88.

Saksena, P., Xu, K., Elovainio, R., \& Perrot, J. (2010). Health services utilization and out-of-pocket expenditure at public and private facilities in low-income countries. World Health Report, $20,20$.

Sangar, S., Dutt, V., \& Thakur, R. (2018). Economic burden, impoverishment and coping mechanisms associated with out-of-pocket health expenditure: analysis of rural-urban differentials in India. Journal of Public Health, 1-10.

Sohrabi, M., \& Tumin, M. (2016). Health issues and challenges among Indian urban poor. Journal of Health Management, 18(3), 460-472.

Turgay, S. (2018). Multi objective simulated annealing approach for facility layout design. International Journal of Mathematical, Engineering and Management Sciences, 3(4), 365-380.

Wang, L. (2018). Big data and IT network data visualization. International Journal of Mathematical, Engineering and Management Sciences, 3(1), 9-16.

Wang, L., \& Alexander, C.A. (2019). Big data analytics in healthcare systems. International Journal of Mathematical, Engineering and Management Sciences, 4(1), 17-26.

Zhang, Y., Berman, O., \& Verter, V. (2009). Incorporating congestion in preventive healthcare facility network design. European Journal of Operational Research, 198(3), 922-935. 